\title{
STUDIES ON CHITOSAN - BENZALDEHYDE INTERACTION IN A HOMOGENEOUS SYSTEM AND ITS ADSORPTION BEHAVIOR OF METAL IONS IN AQUEOUS SOLUTION
}

\author{
Do Truong Thien*, Nguyen Van Tuyen, and Tran Thi Y Nhi \\ Institute of Chemistry, Vietnamese Academy of Science and Technology, \\ 18 Hoang Quoc Viet, Cau Giay, Hanoi, Vietnam
}

Received 28 July 2006

\begin{abstract}
In this work, N-benzylidene chitosan was synthesized by chitosan -benzaldehyde interaction in a homogeneous system. The modified polymers were characterized by means of infrared (IR) spectra, X-Ray diffraction and thermal analysis. Their adsorption behavior was investigated by atomic absorption spectrophotometry. The experimental results showed that N-benzylidene chitosan had better adsorption properties and higher chemical resistance than that of chitosan.
\end{abstract}

\section{INTRODUCTION}

The potential threats associated with the accumulation of heavy metals in various aspect of the environment are generally well recognized. Due to their presence in industrial, mining and agricultural waste streams, heavy metals from point and non-point sources often end up being discharged or washed into surface water during a rain event, where they accumulate in food chain, posing threat to public health and wildlife [3].

Among many methods available for the removal of heavy metals from water namely: chemical precipitation, ion exchange, coagulation, solvent extraction..., adsorption has been shown to be an economically feasible alternative [8]. Chitosan, which is obtained from the deacetylation of chitin, has received considerable interest for removal of heavy metals due to its excellent metalbiding capacity and easy reuse, as well as its relatively low cost and possible biodegradable after usage.

But raw chitosan tend to present some disadvantages such as unsatisfied mechanical properties and high solubility in acidic solution [1]. The focus of this paper is to synthesis N-benzylidene chitosan which is higher chemical resistance than chitosan and to investigate their adsorption capacity of some metal ions.

\section{EXPERIMENTAL}

\subsection{Materials}

- $\quad \beta$-chitin was extracted from squid pen and $\beta$-chitosan was prepared by deacetylation of chitin in alkali solution as reported previously [2]. The degree of acetylation (DA) found by IR was about $10 \%$;

\footnotetext{
* Corresponding author e-mail: thiendt@ vast.ac.vn
} 
- Benzaldehyde, acetic acid, methanol purchased from China were analytical reagent grade.

- $\quad$ The metal salts chosen $\mathrm{Cu}\left(\mathrm{NO}_{3}\right)_{2} .3 \mathrm{H}_{2} \mathrm{O} ; \mathrm{Fe}\left(\mathrm{NO}_{3}\right)_{3} .9 \mathrm{H} 2 \mathrm{O} ; \mathrm{CdSO}_{4} .8 / 3 \mathrm{H}_{2} \mathrm{O}$ were analytical reagent grade. All solutions were prepared with distilled water.

\subsection{Measurements}

Infrared (IR) spectra were recorded on IMPACT-410 Fourier-transformation spectrometer, Germany; X-Ray diffraction patterns were recorded on SIEMENS D5000; Thermo-gravimetric analysis (TGA) of polymers were performed in nitrogen atmosphere on TA-50 thermal analyzer (Shimadzu). The metal ions concentration was determined with a Perkin Elmer atomic absorption spectrophotometer (ASS - 3300).

\subsection{Preparation of $N$-benzylidene chitosan}

$1 \mathrm{~g}$ chitosan was dissolved in $90 \mathrm{ml}$ of aqueous acetic acid $1 \%$ - methanol $(2: 1 \mathrm{v} / \mathrm{v})$ at room temperature in nitrogen atmosphere. Then $1 \mathrm{~g}$ benzaldehyde which had been previously dissolved in $10 \mathrm{ml}$ methanol was added and stirred for 24 hours to obtain a transparent gel. The gel was washed several times with distilled water and methanol to remove any un-reacted benzaldehyde and dried at $60^{\circ} \mathrm{C}$ and gave $1.4 \mathrm{~g}$ of product ( $70 \%$ yield).

\subsection{Adsorption experiments}

$100 \mathrm{mg}$ of chitosan and $N$ - benzylidene chitosan were added to $50 \mathrm{ml}$ salt solution of $\left[\mathrm{Cu}\left(\mathrm{NO}_{3}\right)_{2}, \mathrm{CdSO}_{4}, \mathrm{Fe}\left(\mathrm{NO}{ }_{3}\right)_{3}\right]$, respectively. After stirring at a defined time at room temperature, the mixture were centrifuged and filtered. The metal ion concentration in the filtrate and initial concentration were determined by atomic adsorption spectrophotometry. The adsorption capacity was calculated as follows:

$$
\mathrm{Q}=\frac{\mathrm{V} \times\left(\mathrm{C}_{\mathrm{O}}-\mathrm{C}\right)}{\mathrm{W}}
$$

Where: $\mathrm{Q}$ is adsorption capacity of samples (mmol of metal ions/g of adsorbent); $\mathrm{V}$ is volume of metal ion solution; $\mathrm{C}_{\mathrm{o}}$ is the concentration of the metal ions before adsorption; $\mathrm{C}$ is the concentration of the metal ions after adsorption. $\mathrm{W}$ is weight of chitosan and $\mathrm{N}$-benzylidene Chitosan.
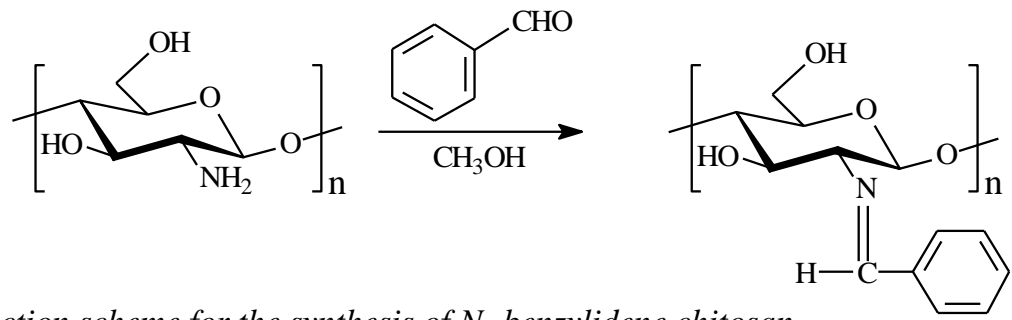

Fig.1: Reaction scheme for the synthesis of $N$ - benzylidene chitosan.

\section{RESULTS AND DISCUSSION}

\subsection{Chitosan - benzaldehyde reaction}

The reaction of chitosan and benzaldehyde occurs by a Schiff's reaction of aldehyde groups 
with amine groups on chitosan chain $[4,7]$. The reaction scheme for the synthesis of $\mathrm{N}$ benzylidene chitosan could be presented as in Fig. 1 .

\subsection{Infrared spectra analysis}

Figure 2 showed the infrared spectra of $N$ - benzylidene chitosan. The intensity of $\mathrm{N}-\mathrm{H}$ stretching at $3471 \mathrm{~cm}^{-1}$. The characteristic peak of $\mathrm{C}=\mathrm{N}$ (imine bond for the Schiff base) stretch vibration appeared at $1643 \mathrm{~cm}^{-1}$ and the peak of mono substituted phenyl at $750 \mathrm{~cm}^{-1}$. All of this evidence supported the grafting of benzaldehyde into chitosan.

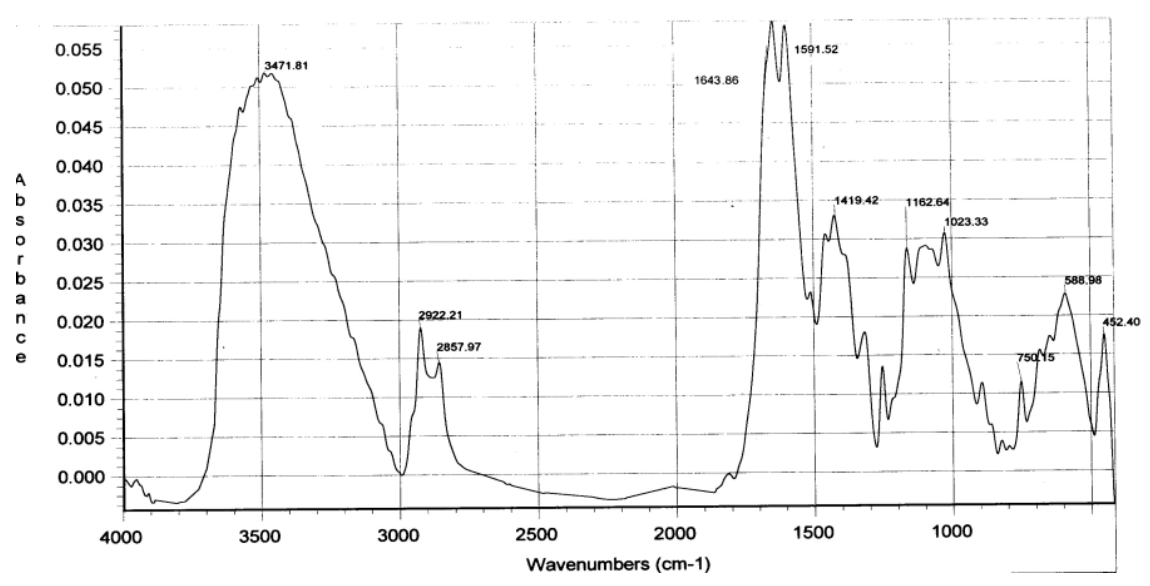

Fig. 2: Infrared spectra of $N$-benzylidene chitosan.

\subsection{X-Ray diffraction analysis}

Figure 3 showed the X-Ray diffraction patterns of chitosan and $N$-benzylidene chitosan. The XRay diffraction pattern of $\beta$-chitosan was indicated by the presence of two peaks at $2 \theta \approx 10^{\circ}$ and $2 \theta \approx 19.8^{\circ}$. For $N$-Benzylidene chitosan, the peak at $2 \theta \approx 10^{\circ}$ almost disappeared and the peak at $2 \theta \approx 19.8^{\circ}$ decreased.

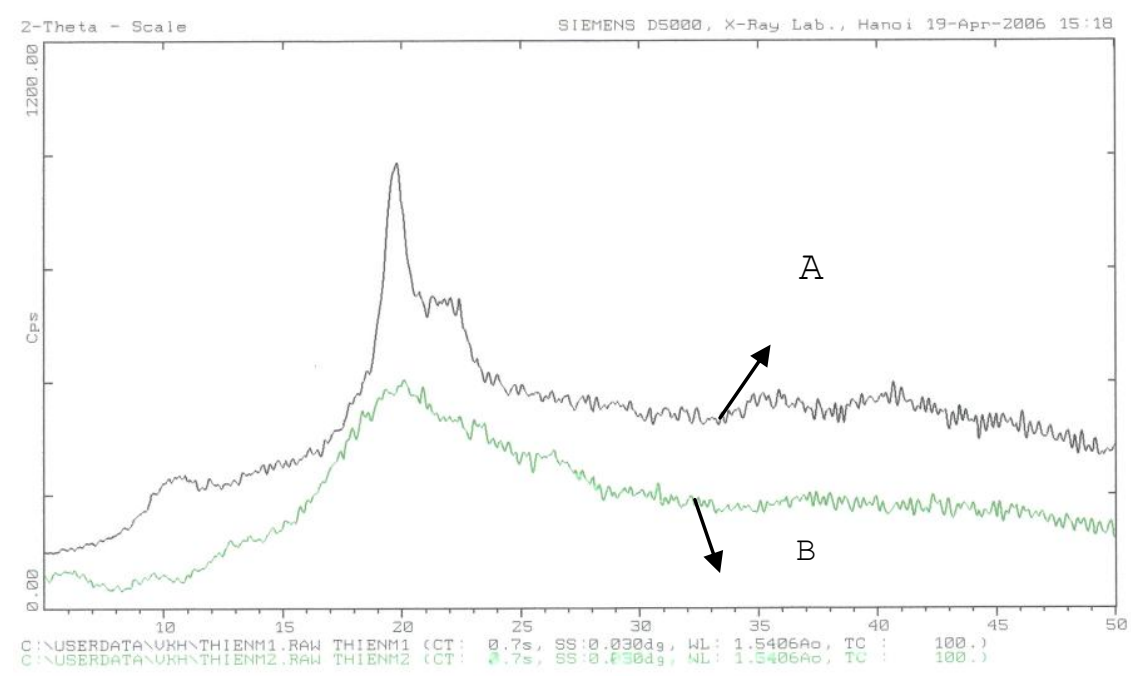

Fig. 3: X-Ray diffraction of chitosan (A) and N-benzylidene chitosan (B). 
The decrease in the crystallinity of $N$-benzylidene chitosan was attributed to the deformation of the strong hydrogen bond in chitosan when amino groups reacts with carbonyl groups of benzaldehyde, indicating that $N$-benzylidene chitosan was more amorphous than chitosan [4].

\subsection{Thermo-gravimetric analysis}

TGA curves of the two polymer were shown in Fig. 4. From about $230^{\circ} \mathrm{C}$ to $355^{\circ} \mathrm{C}$ we could observe the same weight loss for both chitosan and $N$-benzylidene chitosan $(44.5 \%$ and $42.6 \%$ for chitosan and $\mathrm{N}$ - benzylidene chitosan, respectively). The second peak of $N$-benzylidene chitosan at $428^{\circ} \mathrm{C}$ with an additional weight loss of $16.4 \%$ revealed interesting and accurate differences of its thermal behavior in comparison with chitosan. This could be a supported evidence for the reaction between chitosan and benzaldehyde.
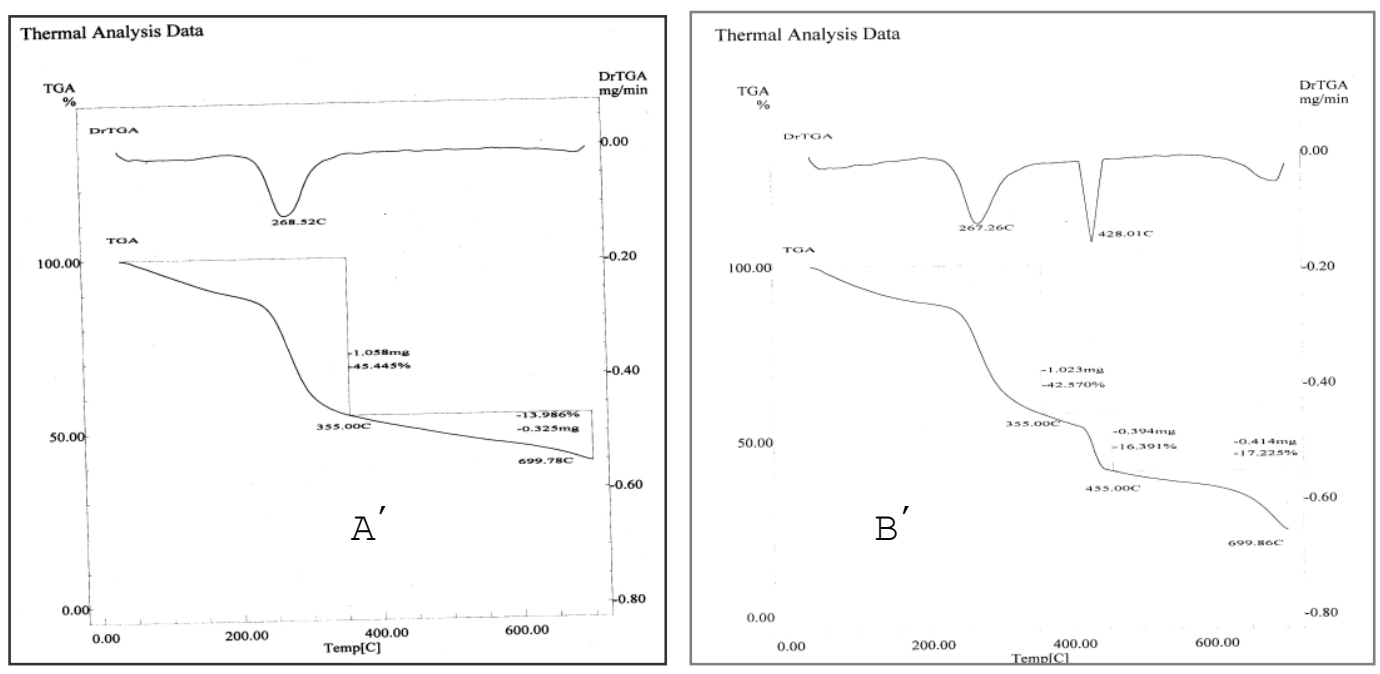

Fig. 4: TGA curves of chitosan (A') and N-benzylidene chitosan (B')

\subsection{Adsorption experiment}

\subsubsection{Effects of agitation period}

As can be seen in the Fig. 5 that the adsorption of metal ions increased rapidly within 3 hours and attained equilibrium at about $6 \mathrm{~h}$ for chitosan (Cts) and 7 hours for $\mathrm{N}$-benzylidene chitosan (CtsB). The adsorption followed the ranking: $\mathrm{Cd}$ (II) $>\mathrm{Cu}$ (II) $>\mathrm{Fe}$ (II). In addition, $N$ benzylidene chitosan has better adsorption properties than that of chitosan. This may be due to CtsB containing aromatic ring and imine bond which are favorable for adsorbing metal cations.

\subsubsection{Effects of $p H$}

The $\mathrm{pH}$ of a solution strongly effects the adsorption capacity of the chitosan and $\mathrm{N}$-benzylidene chitosan. At a low $\mathrm{pH}$, chitosan was soluble, so it is difficult to carry out an adsorption experiment. But for $N$-benzylidene chitosan, which is in gel form, highly solubility in acidic solution is improved. In acidic solution, more protons will be available to protonate the amine groups to form group $\mathrm{NH}_{3}{ }^{+}$, reducing the number of binding sites for the adsorption [8]. The 
figure 6 showed that the adsorption of $\mathrm{Cu}$ (II) increased with increasing $\mathrm{pH}$ of the solution. At $\mathrm{pH}$ value higher than 7, the precipitation occurred simultaneously. The maximum adsorption of ion $\mathrm{Cu}(\mathrm{II})$ was found at $\mathrm{pH} 5.0$.

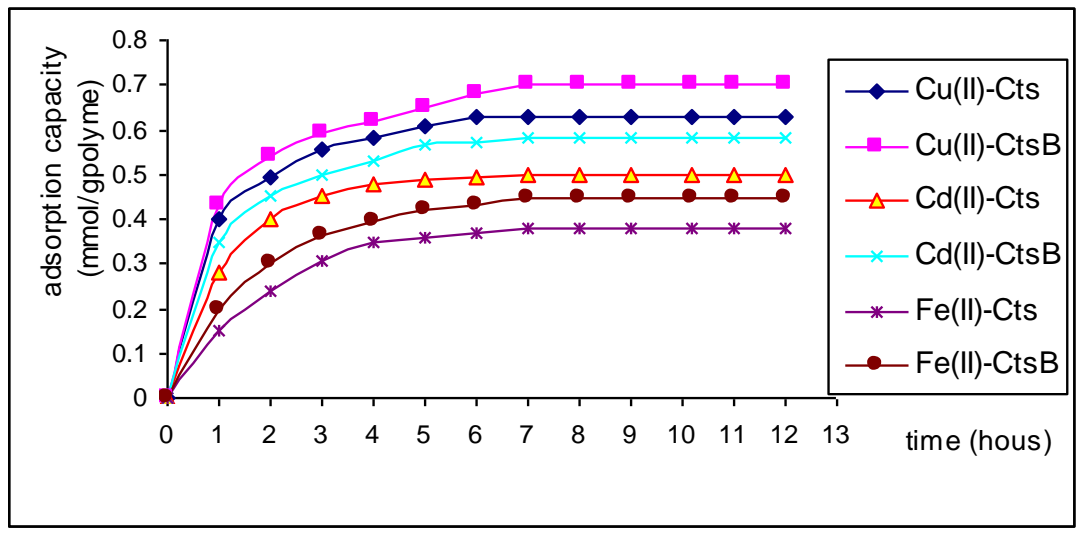

Fig. 5: Effects of agitation period on metal adsorption capacity.

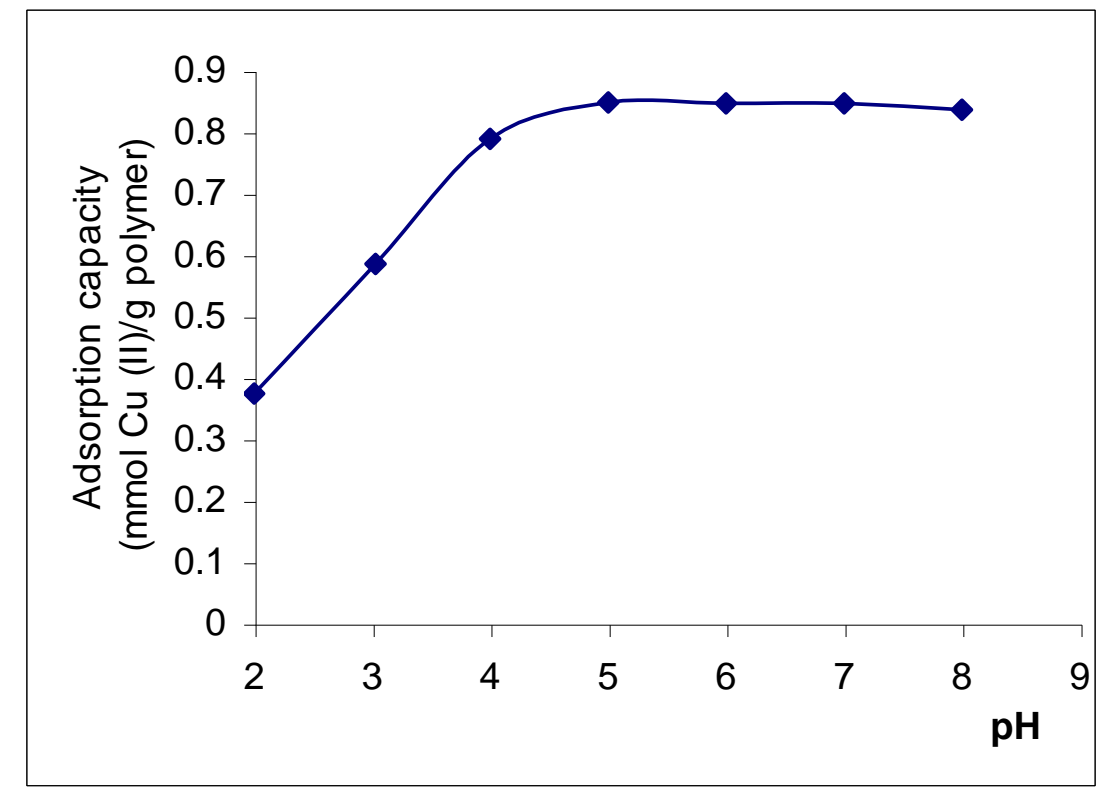

Fig. 6: Effect of pH solution on adsorption capacity.

\section{CONCLUSIONS}

$N$ - benzylidene chitosan was successfully synthesized by the reaction of chitosan and benzaldehyde in a homogeneous system. Its characteristic was investigated by IR, XR and TGA.

Their adsorption properties were also characterized. The results showed that $N$-chitosan benzaldehyde has better adsorption property than that of chitosan and the adsorption followed the ranking: $\mathrm{Cd}$ (II) $>\mathrm{Cu}$ (II) $>\mathrm{Fe}$ (II). 


\section{REFERENCES}

1. Antonio R. Cestari, Uenice F.S. Vieira, and Joana D.S. Matos (2006), Thermodynamic of the $\mathrm{Cu}$ (II) adsorption on thin vanillin-modified chitosan memberance, J. Chem. Thermodynamic, available online at www.sciencedirect.com

2. Do Truong Thien and Pham Thi Bich Hanh (2001), Advances in Natural Science, Vol. 2, pp. 57-68.

3. Eric Guibal (2003), Separation and Purification Technology, Vol. 38, pp. 43-74.

4. Lili Wan, Yuting Wang, and Shahua Qian (2001), J. Appl.Polym. Sci, Vol. 84, pp. 9-34.

5. Martha Ly Arrascue and Hollger Maldonado Garcia (2000), Hydrometallurgy, Vol. 71, pp. 191-200.

6. Oyrton A.C. Monteiro Jr., and Claudio Airoldi (1999), International Journal of Biological Macromolecules, Vol. 26, pp.119-128.

7. Shigehiro Hirano, Kenji Nagamura, Min Zhang, Sun Ki Kim, Byung Geul Chung, Masatoshi Yoshikawa (1998), Carbohydrate Polymer, Vol. 38, pp. 293-298.

8. Wan Ngah, W.S., Ghani, S.Ab., and Kamari, A. (2005), Biosource Technology, Vol. 96, pp. 443-450.

9. Weltrowski, M, Martel, B., and Norcellet, M. (1996), J. Appl. Polym. Sci., Vol. 59, pp. 647654.

10. Yang, Z., Wang, Y., and Tang, Y.J. (1999), Appl. Polym. Sci., Vol. 73, pp. 469-476. 